\title{
VARIABLE CRITERION ANALYSIS OF SIMPLE REACTION TIME DISTRIBUTIONS IN NORMAL, CHIASM-SECTIONED AND CHIASM-CALLOSUM- SECTIONED MONKEYS (Macaca mulatta) ${ }^{1)}$
}

\author{
KiYoko Murofushi
}

\section{Primate Research Institute, Kyoto University}

In split-brain monkeys, the cortico-cortical connections between the cerebral hemispheres are severed. Learning in one hemisphere was disrupted in those monkeys, if the active response systems used during a sensory-guided motor task are strongly lateralized in another hemisphere. For example, if a split-brain monkey is restricted to the use of only one hand during a visual pattern discrimination, it is found on subsequent testing that the contralateral eye-brain is superior to the ipsilateral eye-brain (Trevarthen, 9). Trevarthen (10) has further reported an occurrence of interhemispheric perceptual suppression in a split-brain patient. The capacity of the subjects to speak about general aspects of form and motion for peripheral stimuli confined to the left visual field was contrasted with their failure to give verbal identification of pictures of objects tachistoscopically in the right half of this field. An increase of detection thresholds in the left visual field found during vocalization in normal subjects was similarly explained in terms of interhemispheric visual suppression produced by a lateralized response set (Kinsbourne, 7). Gazzaniga and Hillyard (2) have recently presented evidence, however, which suggests that this disruption of one hemisphere's activities by the other occurs in a process of response

1) This study was supported by a scientific grant of the Ministry of Education in Japan (No.821009, 831037, 1973). The author is grateful to Dr. K. Hara and Dr. K. Kubota for their assistance with surgery, and subsequent histological examination. 
evocation rather than in a perceptual process. To create expectancy of an impending tone and motor response in only one of the surgically separated hemispheres of a human subject, they designed an experiment in which a visual warning signal was presented exclusively to one or the other hemisphere on a given trial. As a result of comparing the mean reaction times (RTs)/ for the different hand-hemisphere combinations, it was found that the RTs for the dominant right hand following the alerting of the right hemisphere were prolonged significantly in relation to those of the other combinations. And these RTs were much longer than even the simple, unwarned RT. However, the contingent negative variation (CNV) during the performance of these $\mathrm{RT}$ s indicated that the warning flashes were being discriminated accurately. The authors have proposed, therefore, that putting a response by the right hand in readiness produces a general inhibition of the competing response system controlled by ipsilateral hemisphere, because the right hand is controlled predominantly from the left hemisphere. Using split-brain baboons, Beaubaton and REQUin (1) have reported that RTs are faster when signals reached the left hemisphere than when the right hemisphere was involved, if the right hand was used. Glickstein ( 3 ) has referred to preliminary evidence, however, that RT was not significantly different when a split-brain rhesus monkey used the ipsilateral eye-hand combination from when the animal used the contralateral combination. Consequently, two questions are presented here to be examined. Is the disturbance in one hemisphere's activities by the other attributed to perceptual suppression or response inhibition? And, is this phenomena found in RT performance by the split-brain rhesus monkeys?

Regarding the first question, theoretical conceptions to distinguish the roles of perceptual process and response variables such as expectancy and motivation have been proposed in psychophysical studies of human perception. For example, GRICE (4) have proposed a decision theory model of stimulus intensity and response evocation. According to this model, stimulus intensity determines the rate of neural events of 
"impulses" arising from a stimulus, whereas a decision criterion or reaction threshold $(\mathrm{T})$ which summarized the state of readiness to respond determines $S$ 's response evocation, or the number of impulses that must be accumulated before a response is made. The time required for the impulse count to reach its criterion values corresponds to $S$ 's $\mathrm{RT}$. The criterion is treated as a normally distributed random variable whose mean (T) and standard deviation $(\sigma)$ are manipulated by a variety of experimental variables and reflect individual differences. Response evocation occurs when the excitatory strength of a response exceeds the momentary value of the criterion. The excitatory strength has been treated as an additive combination of associative or habit strength $(\mathrm{H})$ and sensory strength $(\mathrm{V})$ in conditioning (GRICE, 5). In the situation of simple RT experiment, however, the associative component should be maximal at the beginning of each trial. Thus, the data analysis of RT has been successfully carried in terms of the sensory strength and the response criterion value only (GRICE, 6). Moreover, it has been shown that the recruitment function of sensory strength is negatively accelerated until it reaches criterion, at which point the response occurs.

Thus, the purpose of this study was to determine whether RTs data from monkeys can be interpreted in terms of decision criterion model. Specifically, to distinguish two factors, perceptual and response evocation, which were expected to be involved in a behavioral deficiency by the split-brain monkeys, a variable criterion analysis (GRICE, 6) was applied.

\section{METHOD}

Subjects and surgery: Eight adolescent monkeys (Macaca mulatta) were employed in this study. One monkey served as normal control. Seven monkeys had their optic chiasms sectioned in the midline via a transbuccal approach. Four of them additionally received midsagittal section of the anterior commissure and the corpus callosum. Surgery was performed aseptically under general anesthesia with all of the sectioned structures visualized through an operating 
microscope.

At the conclusion of the experiment one chiasm sectioned (No.208) and three split-brain monkeys (No. 205, 211, 298) were sacrified and perfused with normal saline followed by $10 \%$ formalin. The remaining animals are still tested another experiment and consequently their brains are not available for histological examination. The optic chiasms of four animals were completely cut. In addition, the corpus callosums and anterior commissures of No.205 and No.298 were completely sectioned. In No.211, however, genu corpus callosum remained intact, although the anterior commissure was completely cut.

The monkeys were placed in primate restraining chairs through the experiment and maintained at 90 to 95 per cent of their free-feeding body weights (3 to 4 $\mathrm{kgm})$.

Apparatus, Training, and Trial Events: The subjects were trained in a dimly lit room to await the onset of the first light as a "warning signal" ( $\mathrm{S}^{W}$ ), then to press a lever and hold it down through a variable 0.5 to $2.0 \mathrm{sec}$. foreperiod initiated by the press, releasing it after the occurrence of the second light as a "response signal" $\left(\mathrm{S}^{D}\right)$. Lever release terminated all of lights.

Lever release following $\mathrm{S}^{\mathrm{D}}$ was reinforced by a drop of orange juice from the tube placed in front of monkey's mouth, and counted as a trial. The intertrial interval was $10 \mathrm{sec}$. Lever release during a foreperiod and lever press during an intertrial interval started a new intertrial interval. Trials, stimulus events, and reinforcement contingencies were automatically arranged by a control system presettled depending on the experimental condition. Continuous low-level white noise masked extraneous sounds from the outside.

The stimulus lights were produced by turning on a bulb mounted behind a 1 $\mathrm{cm}$ hole on the stimulus panel which was located $30 \mathrm{~cm}$ from the eyehole panel closely faced the monkey's eyes. Polaroid filters were permanently placed in front of each light on the stimulus panel. During testing another set of polaroid filters was placed in fornt of two eyeholes, oriented such that the stimulus light reached only one eye (Fig. 1). In the control condition, the stimulus light was available for both eyes by turning the orientation of the polaroids in front of the eyeholes to 45 degrees.

The lever was positioned on the right or left side at the height of the animal's hands which were restrained by running the arms through a leather pipe on the waist plate of the chair. Head movement was also restricted by a small box fixed on the neck plate of the chair such that each eye was not placed out of 
the eyehole.

Numbers of reinforcements, responses during the intertrial interval, and lever release during the foreperiod were recorded in the counters. Response latencies for lever release to $\mathrm{SD}^{\mathrm{D}}$ were measured to within 10 milliseconds by an electronic digital counter and punched out on a paper tape for computer analysis.

Procedure for Testing: Monkeys were tested through two stages. Each stage consisted of ten 100-trials sessions, one session per day, lasting about one hour. During the first two sessions, the stimulus light was presented binocularly by setting the polaroid filters in front of the eyeholes at an angle of 45 degrees (Control condition). For the following 8 sessions, monocular vision was used (Experimental condition). Stimulus lights ( $\mathrm{SW}^{\mathrm{W}}$ and $\mathrm{S}^{\mathrm{D}}$ ) were alternately presented in the right or left position in a block of 10 trials (Fig. 1). The first block always started from the side opposite to the hand used in that stage.

During the second stage the same procedure as that of the first stage was repeated except that the other hand was required. The order of hand tested in the first stage was counterbalanced over the subjects (Table 1).

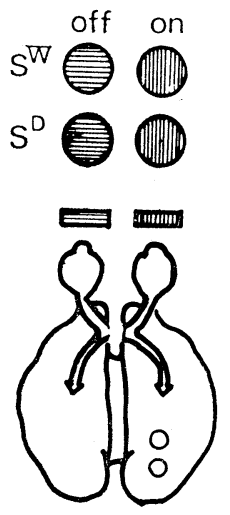

Fig. 1. Schematic representation of visual information available to only one hemisphere in chiasm-sectioned animals. The direction of the striation indicate the orientation of the polaroid filters placed before the visual signals, and before the two eyes of the monkeys.

\section{Results}

Comparisons of Hand-Hemisphere Combinations: Mean RTs and $\mathrm{SD}_{\mathrm{S}}$ for the different eye-hand combinations are shown in Table 1. To examine briefly the differences between the test conditions, an analysis of variance was done by the two factors of hands and eyes for each subject. Significant difference between hands were obtained for $6 \mathrm{Ss}$, which are marked with ${ }^{*}$ in Table 1 . All of these cases indi- 
Table 1. Reaction times in msec (SD) for lever press to visual signals in eye-hand combinations

\begin{tabular}{|c|c|c|c|c|c|c|}
\hline \multirow{2}{*}{\multicolumn{2}{|c|}{ Ss }} & \multirow{2}{*}{ Hand } & \multicolumn{3}{|c|}{ Visual Signals to } & \multirow{2}{*}{$\begin{array}{l}\text { Hand in } \\
\text { First Stage }\end{array}$} \\
\hline & & & Both eyes (200) & $\mathrm{R}$ eye $(400)$ & L eye $(400)$ & \\
\hline \multirow[t]{2}{*}{ No. 213} & \multirow[t]{2}{*}{ (Normal) } & $\mathrm{R}_{*}$ & $294.1(53.3)$ & $360.2(199.2)$ & $372.4(198.5)$ & \multirow[t]{2}{*}{$\mathrm{R}$} \\
\hline & & $\mathrm{L}$ & $367.5(119.9)$ & $301.3(60.5)$ & $306.9(59.7)$ & \\
\hline \multirow[t]{2}{*}{ No. 214} & \multirow[t]{2}{*}{ (Chiasm) } & $\mathrm{R}_{*}$ & 453. $2(191.6)$ & $465.6(509.8)$ & $465.9(326.9)$ & \multirow[t]{2}{*}{$\mathrm{R}$} \\
\hline & & $\mathrm{L}$ & $298.6(83.6)$ & $314.6(115.4)$ & $307.3(118.0)$ & \\
\hline \multirow[t]{2}{*}{ No. 388} & \multirow[t]{2}{*}{ (Chiasm) } & $\mathrm{R}_{*}$ & $445.0(131.1)$ & $383.2(142.6)$ & $448.8(180.2)$ & \multirow[t]{2}{*}{$\mathrm{L}$} \\
\hline & & $\mathrm{L}$ & $486.8(264.1)$ & $404.6(215.7)^{* *}$ & $578.7(458.9)$ & \\
\hline \multirow[t]{2}{*}{ No. 208} & \multirow[t]{2}{*}{ (Chiasm) } & $\mathrm{R}_{*}$ & $270.1(125.6)$ & $1046.0(1283.7)$ & $405.8(283.2)$ & \multirow[t]{2}{*}{$\mathrm{R}$} \\
\hline & & $\mathrm{L}$ & $415.7(185.4)$ & $378.1(139.3) * *$ & $369.4(130.5)$ & \\
\hline \multirow[t]{2}{*}{ No. 205} & \multirow[t]{2}{*}{ (Split) } & $\mathrm{R}_{*}$ & $454.2(625.7)$ & $1014.1(1924.3)$ & $475.4(387.4)$ & \multirow{2}{*}{$\mathrm{R}$} \\
\hline & & $\mathrm{L}$ & $490.2(240.0)$ & $409.0(148.5)^{* *}$ & $441.3(242.3)$ & \\
\hline \multirow[t]{2}{*}{ No. 298} & \multirow[t]{2}{*}{ (Split) } & $\mathrm{R}_{*}$ & $450.4(107.0)$ & $483.1(266.2)$ & $445.9(319.2)$ & \multirow[t]{2}{*}{$\mathrm{L}$} \\
\hline & & $\mathrm{L}$ & $523.7(208.5)$ & $559.6(375.3)$ & $562.7(333.2)$ & \\
\hline \multirow[t]{2}{*}{ No. 211} & \multirow[t]{2}{*}{ (Split) } & $\mathrm{R}$ & $389.0(259.1)$ & $574.8(339.2)$ & $677.7(472.6)$ & \multirow[t]{2}{*}{$\mathrm{R}$} \\
\hline & & $\mathrm{L}$ & $469.5(100.0)$ & $484.0(168.8)^{* *}$ & $827.8(538.0)$ & \\
\hline \multirow[t]{2}{*}{ No. 309} & \multirow[t]{2}{*}{ (Split) } & $\mathrm{R}$ & $514.9(148.0)$ & $708.9(474.2)$ & $554.2(318.0)$ & \multirow[t]{2}{*}{$\mathrm{L}$} \\
\hline & & $\mathrm{L}$ & $590.0(233.7)$ & $689.0(315.2)^{* *}$ & $663.5(405.5)$ & \\
\hline
\end{tabular}

cate that RTs in the second stages were faster than those in the first stage, which may reflect the effect of practice in observing monocularly. If a significant interaction effect by hand and eye was obtained, then each individual mean was compared with others. As a result of this analysis, eye and hand interaction effects were found in $5 \mathrm{Ss}$ as indicated in Table 1 with ${ }^{* *}$ marks. These interaction effects can be classified into two types as shown in Fig. 2. In the first type shown in left panel of Fig. 2, when the right hand was used, RTs are longer if signals reached the right hemisphere than if the left hemisphere was involved $(p<.001)$. This difference between hemispheres does not 
appear for the left hand. Three subjects are included in this type, one chiasm-sectioned and two chiasm-callosum-sectioned animals, all of which showed right hand preference determined by a test in which the first hand to pick up a piece of potato was recorded. In the second type shown in the right panel of Fig. 2, when signals reached the right hemisphere $\mathrm{RT}_{\mathrm{s}}$ are faster than when the left hemisphere was involved for both hands $(p<.001)$. This difference between hemispheres, however, is significantly greater when the left hand performed the response than when the right hand was used $(p<.001)$. One chiasmsectioned animal which had left hand preference and one chiasmcallosum-sectioned $S$ of which hand preference was not clear are involved in this type of interaction between hemisphere and hand.

Since the difference between variances of RTs in four combinations of hand and hemisphere is quite large as appeared in Table 1, an analysis of variance is not appropriate, nor does it provide sufficient information to know how differently are distributed $\mathrm{RT}_{\mathrm{S}}$ in the various hand-hemisphere combinations. Patterns of distribution for each subject are investigated in terms of the cumulative probability curves as a function of the time from signal onset for each condition shown in Fig. 3. At least three points concerning the eight subjects' cumulative distributions should be noted. The first, for the subjects which showed significant difference only between hands, it is found that two curves in the slower hand almost parallely shifted to the right in the abscissa (No. 213 and No. 214). The second, most striking feature in the distribition of RTs by operated subjects is that the RTs longer than one second appeared about 15 to $20 \%$ in the ipsilateral hand-hemisphere combination which significantly differed from other conditions (No. $388,208,205,211,309)$. Even in the subject which did not show significant interaction effect between hand and hemisphere (No.298), the same tendency was observed. These unusually prolonged tail of RT's distrubution may reflect failure of signal detection. The third, turning eyes to the opposite tail of distribution, an early rise was noticed in the right hand regardless of the remaining RT's rate (No.208 and 


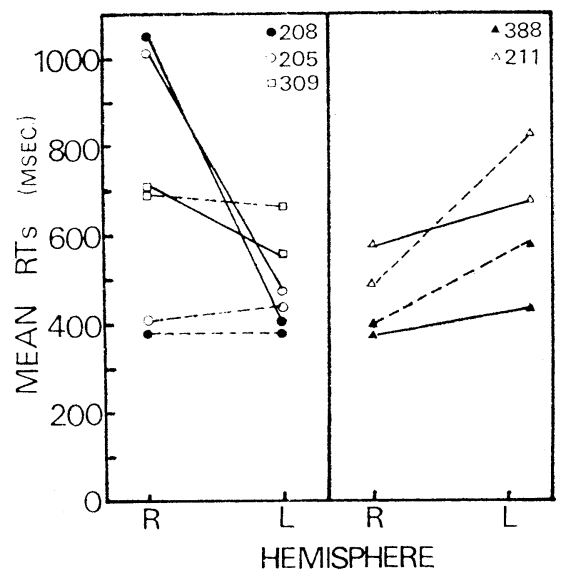

Fig. 2. RT's differences in terms of hand-hemisphere combinations. The right hand and left hand are repsesented by solid and broken line, respectively.
No. 309).

All above results lead to one conclusion that there is some evidence to indicate the prolonged R'Ts in the ipsilateral hand-hemisphere combination compared with RTs in the contralateral combination. This effect, however, was obtained not only from splitbrain monkeys, but also from chiasm-sectioned monkeys. Moreover, this effect can be illustrated not only by the mean RT which became significantly longer, but by the different pattern of distribution.

What causes these differences in RTs is the question addressed in the following data analysis.

Comparisons of Decision Criterions: According to GRICE's model $(\mathbf{5}, \mathbf{6})$, the first step in the analysis was to transform the cumulative proportions in Fig. 3 to their corresponding normal deviates. In this form, the transformed cumulative distribution now provide estimates of the growth of excitatory strength (E) following signal onset. Any point on one of the function estimates the distance of the sensory recruitment function $(\mathrm{V})$ at that time to the mean of criterion distribution (T) in units of the standard deviation $(\sigma)$ of that distribution.

That is, each point estimates $\mathrm{E}=\mathrm{V}-\mathrm{T}$. To make a common scale across the conditions within a subject as well as between subjects, the next step is to estimate the distances between $\mathrm{T}$ s in the conditions and subjects and those relative sizes of $\sigma \mathrm{s}$. It involves plotting for adjacent conditions which are called response evocation characteristics (RECs) by GRICE, and which is similar to ROC in signal detection 
Murofushi : Reaction Time Distributions in Split-brain Monkeys
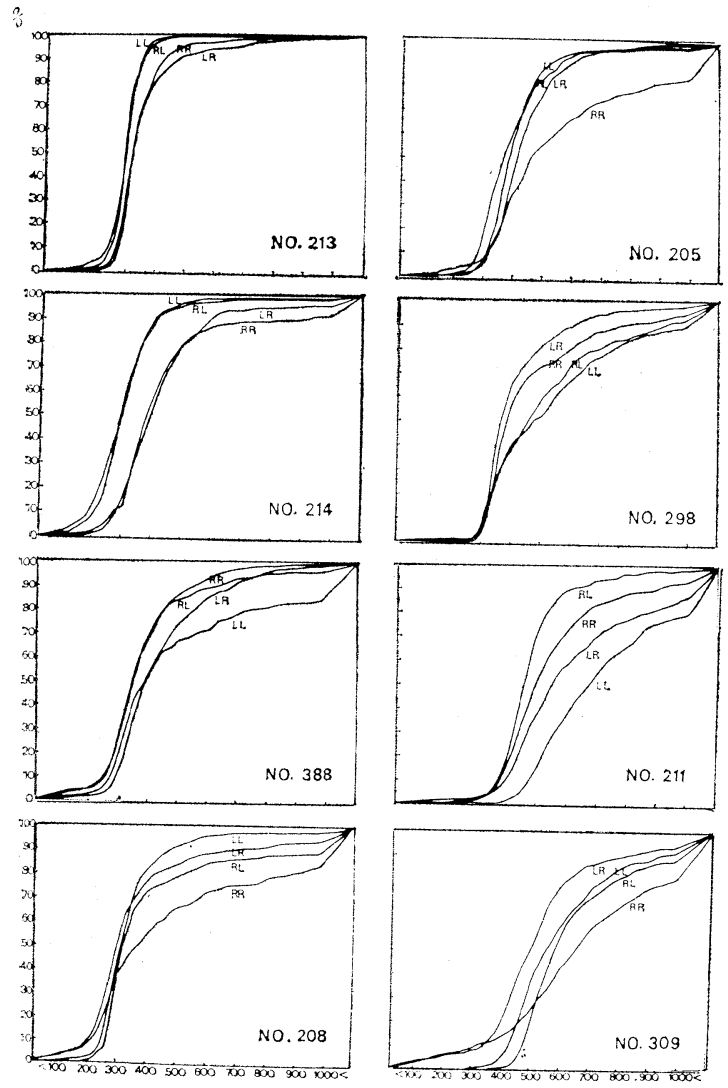

mSCC

$1 \mathrm{~s} \leqslant \mathrm{C}$

Fig. 3. Cumulative RT distribution functions for each condition in each subject.

analysis. All RECs used in this study are presented in Fig.4. An REC is a plot of probability of response for one eye-hand combination against probability for a second combination on normal-normal coordinates. Ten points, each point representing one 20 -msec. class interval, were plotted for within-S scaling between eye-hand conditions, which are represented in every three plots from the left in a row in Fig. 4. The forth plots in each row relate $S s$ No.214, 388, 208, 205 to No. 213 
for the R-L eye-hand combination. The lower two Ss No.211 and 309 are plotted against No.205 instead of No. 213, because overlapping portions with No. 213 which are available for such a plot were too small to satisfy the linearity. The lines were fitted by the method of the least square. The general picture suggests rather good overall support for the assumption of normal distribution, although some

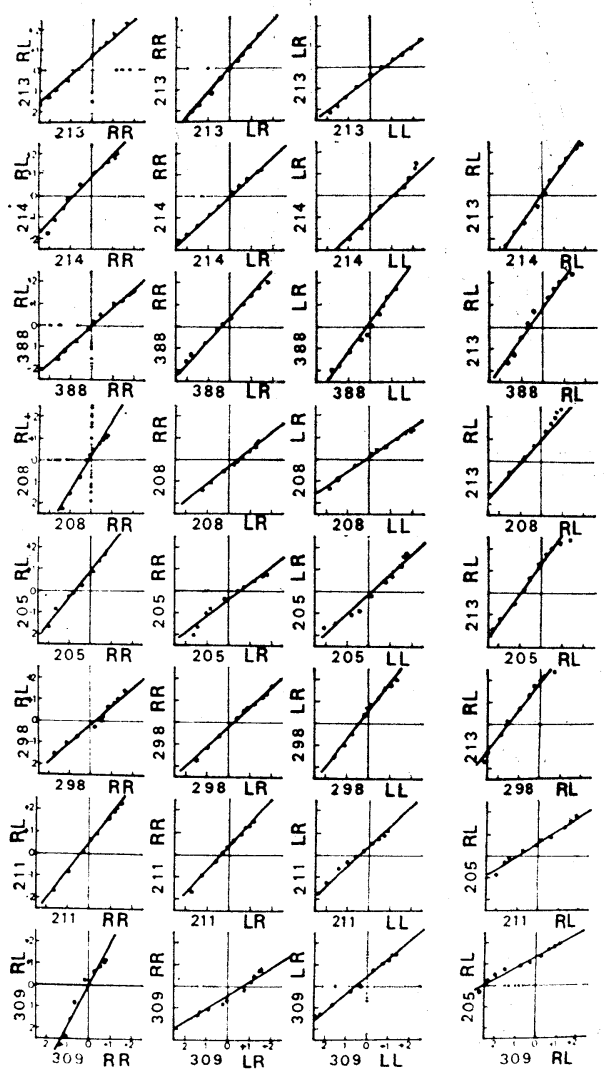

Fig. 4. RECs used in scaling solution. The units are normal deviates corresponding to proportions in the cumulative distribution. 


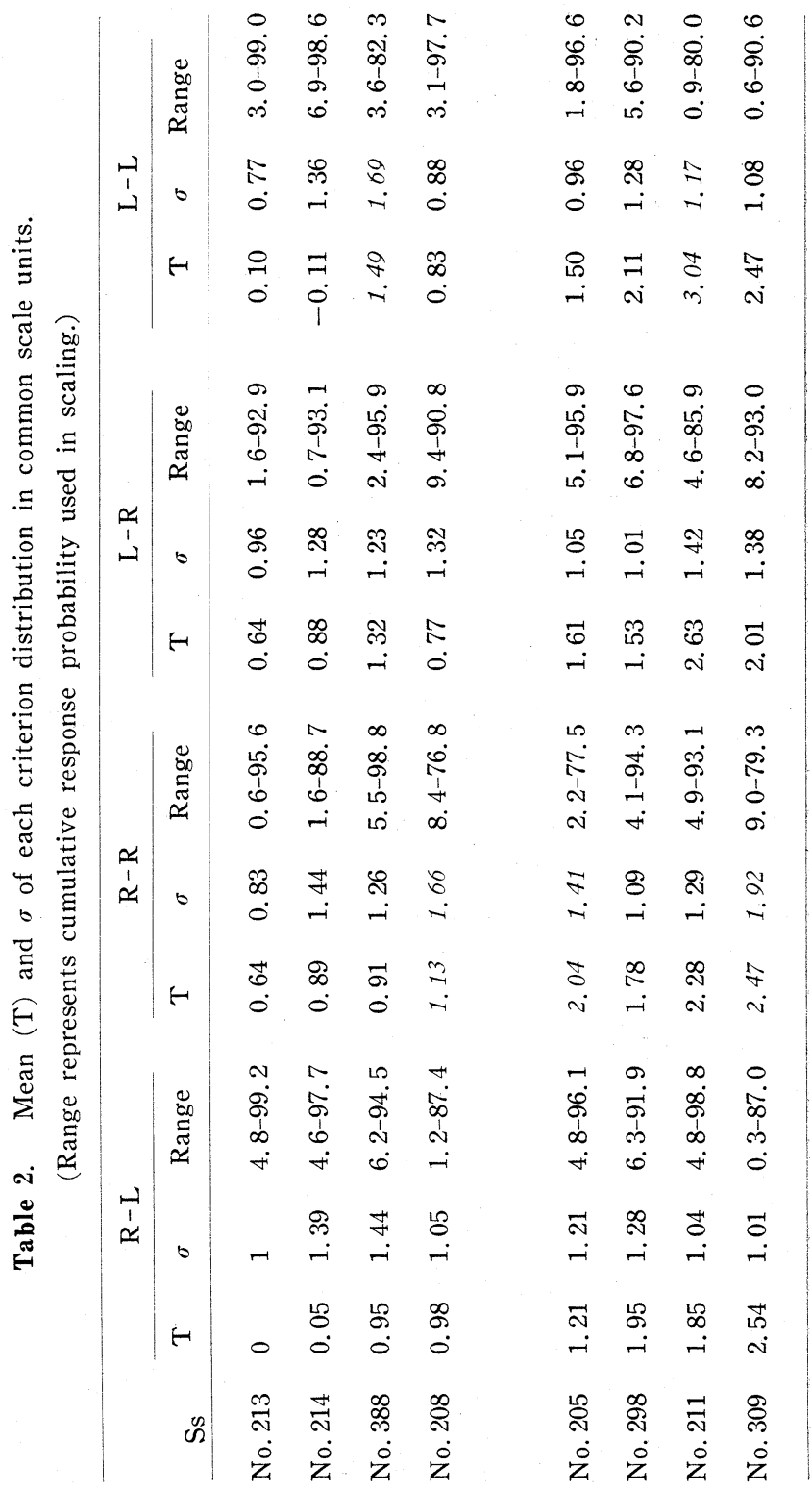


departures from linearity are perceived. The slope of the line is the ratio of the standard deviations $\sigma_{\mathrm{x}} / \sigma_{\mathrm{y}}$. The y-intercept is the distance (d) between the means of two criterion distributions $\left(\mathrm{T}_{\mathrm{S}}\right)$ in units of $\sigma_{\bar{y}}$, and the $\mathrm{x}$-intercept is $\mathrm{d}$ in units of $\sigma_{\mathrm{x}}$. In the first plot of No. 213, for example, the $y$-intercept is +0.644 and the slope is 0.828 . This represents that the criterion mean in $\mathrm{R}-\mathrm{R}$ condition is 0.644 higher than that in $\mathrm{R}-\mathrm{L}$ condition, although the standard deviation is smaller in $\mathrm{R}-\mathrm{R}$ rather than that in $\mathrm{R}-\mathrm{L}$. In the third plot in the same subject, on the other hand, the $y$-intercept is -0.542 and the slope is 0.796 , which means that the $S$ reacted in $\mathrm{L}-\mathrm{L}$ condition with a criterion distribution of which the mean is lower and the standard deviation is smaller than those in $\mathrm{L}-\mathrm{R}$ condition. These two figures well illustrate that No. 213 performed faster when the left hand was used than when the right hand was required. As regards the comparison between subjects, it is demonstrated that No. 213 always performed with lower mean and smaller standard deviation of the criterion distribution than other animals did. Thus, if the mean criterion in No.213's R-L condition is arbitrarily chosen as origin and its $\sigma$ as the unit of scaling, then all remaining $\sigma \mathrm{s}$ and all values of $\mathrm{d}$ can be sequentially converted to the common unit. The resulting values of $\mathrm{T}$ and $\sigma$ for every condition of each subject in this common scale are presented in Table 2. As expected, in the $S s$ which significantly showed interaction effects between hand and hemisphere, its highest mean appears in one of the ipsilateral combinations as indicated in italics in the Table 2, except for No.309. In this subject, when the right hand was used, a quick rise in the short RTs was observed in the cumulative response probability of Fig. 2 as mentioned before. This tendency resulted in the low criterion mean on the right hand of No.309. The same effect also functioned upon the R-R condition of No. 208 in the direction to lower its criterion value. Therefore, these estimates of response criterion value do not provide an adequate account of the different RT caused by the interaction effects between hand-hemisphere within subject.

A remarkable difference, however, was obtained when the normal 


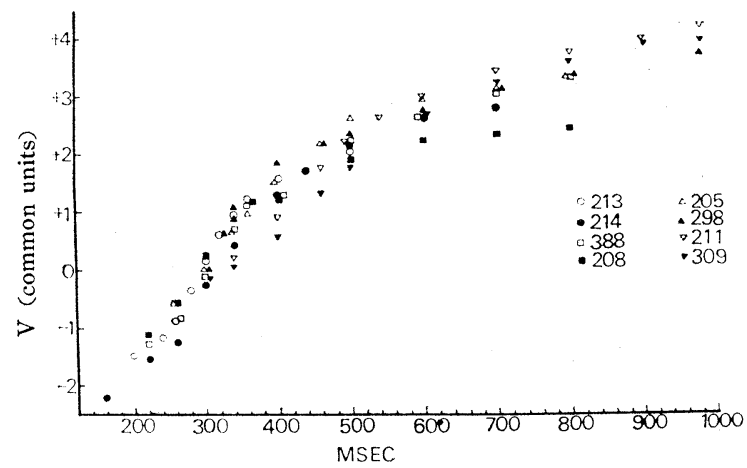

Fig. 5. Estimates of the sensory recruitment function on the common scale.

and 3 chiasm-sectioned animals as a group were compared with 4 chiasm-callosum sectioned ones for the mean criterion values ( $T$ ) in a common scale $(p=0.014$ in $U$ test). This means that the split-brain monkeys performed visual simple reaction task with higher criterion for response evocation than that in normal and chiasm-section monkeys.

Estimation of Sensory Recruitment Function: The next step in the analysis is to convert each of the transformed cumulative distribution functions to the common scale. This is accomplished by multiplying each value by the appropriate $\sigma$ and adding the value of $T$. That is, this procedure permits one to estimate sensory recruitment function for $\mathrm{V}$, which was assumed the same for all conditions and all subjects. To examine this hypothesis, a single estimate for the function on each subjects has been obtained by computing the mean of four separate estimates from each eye-hand combination at the same 20 -msec class interval. These means are plotted in Fig. 5, and describe one negatively accelerated funtion across the eight subjects, although partly deviations are found in No. 208 and 309. This exponential growth function is compatible with that cbtained by the normal human subjects (GRICE, 5). If the means of the criterion distribution are located along the ordinate in Fig. 5 at positions indicated in Table 2, the entire model 
may be understood by implying that the cumulative probability of responses at any time, $t_{i}$, is the proportion of the criterion distribution below the sensory recruitment function at $t_{i}$.

\section{Discussion}

The first main result obtained in this experiment was that prolonged $\mathrm{RT}$ s in the ipsilateral hand-hemisphere combination were found in chiasmsectioned monkeys as well as in chiasm-callosum-sectioned monkeys. Moreover, compared with other conditions, a peculiar feature of the distribution of $\mathrm{RT}_{\mathrm{S}}$ in those conditions was described by the portion of the extreme tail longer than one second which reached to almost $20 \%$ of all RTs. Since the hand used in this simple RT task was restricted to only one side during one stage of experiment which lasted ten days, the response system in this situation should be strongly lateralized as noticed by GazzAniga and Hillyard ( 2 ). Therefore, these phenomena would be attributed to an interference by the dominant contralateral control system, as expected at the beginning of this experiment. The findings also indicates, however, that the independent brain disconnected at the cortico-cortical level is not critical for this phenomena but the most substantial aspect is that the visual signal reached to only one hemisphere, because the chiasm-sectioned animals showed the same effects as it in the split-brain monkeys. Furthermore, a significant difference of response criterions between chiasm-sectioned and split-brain monkeys was found. This means that the findings not only accounted for the process of response evocation but also for perceptual process, which suggested by the previous studies in the human subjects (Kinsbourne, 7; Trevarthen, 10) as an interhemispheric visual suppression produced by the lateralized response set. Unusual proportions of RTs longer than one second in these ipsilateral condition also give a support for this view, because it may reflect a failure of signal detection.

Thus, our results of analysis implicate two components which are involved in the prolonged RTs by the split-brain monkeys. One is 
related to the process of visual detection and the other to the process after detecting visual signals to producing the response. The former is manifested by the operation to send visual information into only one hemisphere when the use of hand is restricted. It produced a deficiency of performance when the ipsilateral eye-hand combination was used in the chiasm-sectioned and chiasm-callosum-sectioned monkeys. The latter participates in raising the threshold to emit the response by the operation to disconnect two hemispheres when the use of eye and hand is restricted. It resulted in the delayed RTs performed by chiasmcallosum-sectioned monkeys.

The operation of disconnecting two hemispheres itself, however, did not produce the same effects if the use of eyes was not restricted. The data in the control condition (Table 1) showed that binocular vision performed faster than monocular vision by the first hand, but became slow by the second hand in the disconnected brain (No. 205 and 211). These facts induce the following inference. The first, when two independent hemispheres received the same information, they functioned cooperatively and made RTs faster than when only one hemisphere received information. Then, after having an experience that information reached only one hemisphere, two hemispheres tended to act independently or might have an interference, even if they get the same information simultaneousely, and resulted in slow $\mathrm{RT}_{\mathrm{s}}$. In the second group in which the left hand was used in the first stage (No.298 and 309), these effects were not observed. Because, the two stages were separated by the period more than three weeks in this group, instead of five days in the first group, to avoid such an interference. This suggests that two disconnected hemispheres were interacting facilitatively for some conditions and inhibitory for others in the performance of RT tasks.

There are other problems provided by the results of this experiment, and remained unsolved at present data analysis. The first problem, the deficiency appeared in the ipsilateral hand-hemisphere combination was found only with one hand in each subject, only the right hand in three and only the left hand in two animals. These inconsistencies 
among subjects are possibly examined as a result of two variables, one is experience effects in monocular vision and the other is hand preference. However, neither possibility could explain these results when considered independently. In the previous studies, similar deficiency in the ipsilateral hand-hemisphere combination has been found only in the right hand, when human (GAZzANigA and HillyARD, 2) and baboon (Beaubaton and Requin, 1) were used as the subjects. A functional asymmetry between hemispheres after callosal section has been reported by Kulics et al. ( 8 ) for macaques in go, no-go discrimination tasks.

The second problem, correspondence between histological and behavioral findings was not clear from this study. If it is assumed that significant ipsilateral deficiency was resulted from a section of the optic chiasm, the data obtained from two monkeys, split-brain (No. 298) and ostensibly chaism-sectioned (No.214) are not consistent with it. Moreover, one subject (No.211) with an intact genu corpus callosum, showed significant behavioral deficiency just like another complete split-brain monkey. The behavioral function of this part of corpus callosum has not been identified.

\section{SUMMARY}

Normal, chiasm-sectioned, split-brain monkeys ( $M$. mulatta) were binocularly trained to press a lever after onset of a visual warning signal and to release it after various forperiods terminated by a second visual signal. The use of hand was restricted to one side. After training, they were monocularly tested in each of the eye-hand combinations. When ipsilateral eye-hand combination was used, significantly prolonged reaction times ( $\mathrm{RTS}$ ) were found in two of three chiasmsectioned and three of four split-brain monkeys, but in different hands. The distribution of RTs in these conditions was characterized by a group of extremly long $\mathrm{RT}$ s. Data were represented in the cumulative probability distributions, which were used to estimate the means and $\sigma$ of the response criterion by an application of variable criterion model. Mean criterions of the split-brain monkeys were significantly 
higher than those of the chiasm-sectioned monkeys, which were not different from that of the normal monkey. Finally, an exponential growth function of sensory recruitment to these different criterions was demonstrated on a common units across the eight subjects. The results of these analysis imply that two components were involved in the delayed RTs appearing in these split-brain monkeys. One is related to the process of signal detection and the other to the process of response evocation.

\section{REFERENCES}

1. Beaubaton, D. and Requin, J. The time course of preparatory process in split-brain monkeys performing a variable foreperiod reaction time task. Physiol. and Behav., 1973, 10, 725-730.

2. Gazzaniga, M.S. and Hillyard, S. A. Attention mechanisms following brain bisection. In A.F.SAnders (Ed.) Attention and Performance IV. Amsterdam: North Holland Publ. 1973, 221-238.

3. Glickstein, M. Brain mechanisms in reaction time. Brain Res., 1972, 4O, 3337.

4. Grice, G. R. Stimulus intensity and response evocation. Psychol. Rev., 1968, 75, 359-373.

5. Grice, G. R. Decision theory of response evocation. In G. H. Bower (Ed.) The Psychology of Learning and Motivation. New York:Academic Press, 1972, 1-65.

6. Grice, G. R. Application of a variable criterion model to auditory reaction time as a function of the type of catch trial. Perception and Psychophysics, 1972, 12, 103-107.

7. Kinsbourne, M. The cerebral basis of lateral asymmetries in attention. Acta Psychologica, 1970, 33, 193-201.

8. Kulics, A. T., Carlson, K. R., and Werner, G. Signal detection analysis of stimulus discrimination in normal and split-brain monkeys, Brain Res., 1974, 81, 119-132.

9. Trevarthen, C. Double visual learning in split-brain monkeys. Science, 1962, 136, 258-259.

10. Trevarthen, C. Experimental evidence for a brain-stem contribution to visual perception in man. Brain Behav. Evol., 1970, 3, 338-352. 